\title{
CURRENT TRENDS IN PRESCRIBING ANTIMICROBIAL DRUGS ON PATIENTS SUFFERING FROM ENT INFECTION IN A TEACHING HOSPITAL IN KHULNA
}

\author{
ISLAM B ${ }^{1}$, PAUL MK ${ }^{2}$, SWEETY AA ${ }^{3}$, FARHAD M ${ }^{4}$, AHMED F ${ }^{5}$, NIZHU LN ${ }^{6}$, BEGUM K ${ }^{7}$, \\ CHOWDHRY IA ${ }^{8}$, RAHMAN HH ${ }^{9}$
}

\begin{abstract}
:
Background: Inappropriate use of antimicrobials results in development of resistance to antibiotics.

Aims and objectives: The present study was aimed at evaluating the current trends in prescribing antimicrobials in patient sufferings from ENT infection.

Methods: A descriptive cross sectional study was carried out at ENT out-patient department of Khulna Medical College Hospital for three months in which data were collected from 500 prescriptions in customized proforma and analyzed using the World Health Organization (WHO) prescribing indicators.

Results: Majority of the patients were females (55\%) which was commonly seen between the age group of 15-24 years (39.2\%). The most common diseases reported was Recurrent tonsillitis (16.4\%) followed by Pharyngitis (15\%), CSOM (13.2\%). Commonly prescribed oral antimicrobials were a-lactams(39.72\%) followed by fluroquinolones $16.16 \%$ and macrolides(4.98\%). Among the a-lactams, Cefuroxime+clavulenic acid(20.39\%) was more used followed by amoxicillin+clavulanic acid(15.55\%), and among fluroquinolones was ciprofloxacin $9.81 \%$ and among macrolides was azithromycin(4.98\%). 57.70\% patients received single antimicrobials whereas $42.3 \%$ received multiple antimicrobials. Average number of antimicrobials prescribed per patient being 1.32. Concomitant therapy included antihistamines(22.93\%), Nasal decongestant(21.56\%). Average number of drugs per prescription was 3.07. All drugs were prescribed in brand names. Oral and topical preparations were frequently prescribed rather injection prescribed was nil. Percentage of encounters with an antibiotic prescribed was100\%. and only 28..72\% drugs were prescribed from Essential Drug List of Bangladesh 2008.
\end{abstract}

Conclusion: Prescribing multiple antimicrobials in this study without definite culture and sensitivity reports make the study less rational in accordance with the standard guidelines.

Key words: antimicrobials, ENT drugs, rational prescribing.

J Dhaka Med Coll. 2018; 27(2) : 155-160

\section{Introduction}

Diseases of the ear, nose and throat (ENT) affect the functioning of adults as well as children, often with significant morbidity causing impairment of routine life of affected patients. ${ }^{1}$ Infectious diseases are among the commonest causes of morbidity and mortality in most developing countries of the world. ${ }^{2}$ According to the world health report of 2010, it has been estimated that respiratory infections were the fourth major cause of mortality which is responsible for global number of deaths and on other hand it also generated that 94.6 disability adjusted life years lost worldwide 3,4,5,6. According to a recent study, acute respiratory infections are the most frequent reason for seeking medical attention and are the reason for $75 \%$ of the antibiotic prescriptions each

1. Dr. Baishakhi Islam, Assistant Professor, Department of Pharmacology \& Therapeutics, Gazi Medical College, Khulna.

2. Dr. Mitun Kumar Paul, Assistant Professor, Department of ENT, Gazi Medical College Hospital, Khulna.

3. Dr. Afroza Akbar Sweety, Assistant Professor, Department of Virology, Dhaka Medical College, Dhaka, Bangladesh

4. Dr. Mohammad Farhad, Medical Officer, DGHS, Dhaka

5. Dr. Farhana Ahmed, Registrar, Department of Cardiology, NICVD, Dhaka

6. Dr. Lutfun Nahar Nizhu, Specialist ICU, Square Hospital, Panthpath, Dhaka

7. Dr. Khadija Begum, Associate Professor of Medicine, Ad-din Women's Medical College, Dhaka

8. Dr. Iqbal Ahmed Chowdhury, Assistant Professor of Medicine, Colonel Malek Medical College, Manikganj

9. Dr. Hasan Hafizur Rahman, Consultant, Medicine, NIKDU, Dhaka

Correspondence: Dr. Baishakhi Islam, Assistant Professor, Department of Pharmacology \& Therapeutics, Gazi Medical College, Khulna, Mobile no. 01715113346, email: drbaishakh@gmail.com)

Received: 12 May 2018

Revision: 26 August 2018

Accepted: 08 September 2018 
year. ${ }^{5}$ These ENT infections are generally caused by microorganisms viz bacteria, fungi and viruses and accordingly the treatment involves the use of appropriate antimicrobials ${ }^{7}$.In present scenario antibiotic is a highly misused medicine. $^{3}$

Irrational use of antimicrobial agents is a great public health concern because of its increased chances of development of antibiotic resistance in a community ${ }^{4.8}$. This in turn gives rise to the need of scrutinizing the antibiotic prescription pattern of the physicians, so as to ensure judicious use of antimicrobial agents. ${ }^{4}$ Drug utilization studies aids in commenting about unnecessary and irrational prescribing which increases burden of cost of therapy, also causes loss of working hours (either due to hospitalization or morbidity). ${ }^{9}$ Therefore, it is imperative to evaluate and monitor the drug utilization patterns from time to time, to enable suitable modifications in prescribing patterns to increase the therapeutic benefit and decrease the adverse effects to optimize the medical services for the patients. ${ }^{3}$ The ultimate aim of the antimicrobial drugs therapy is to eradicate infections and minimize the morbidity and the complications associated with them. ${ }^{7}$

\section{Methods:}

A cross-sectional descriptive study was conducted to evaluate the trend in prescribing antimicrobials on patients suffering from ENT infection in Khulna Medical College Hospital over a period of three months (from January to March 2018) after taking proper approval from hospital administration. A total of 500 patients attending in the ENT OPD during the study period were enrolled in the study on the basis of inclusion and exclusion criteria. Patients of all age group for both sexes and who willing to participate in the study were included in this study. However indoor patients, follow up visit patients and patient with co morbid diseases like DM, HTN etc. patients who were not treated with antimicrobials and who will not willing to participate in the study were excluded from this study. Data were collected in preformed customized pro-forma in the form of questionnaire and from physician's prescription which is analyzed on the basis of standard guideline. Compilation and analysis of data were done by using Microsoft Excel 2007 and represented as number and percentage.
The present study followed some WHO prescribing indicators which are as follows: (1)Average number of drugs per encounter (2)Percentage of drugs prescribed by generic name (3)Percentage of encounters with an antibiotic prescribed(4)Percentage of encounters with an injection prescribed (5)Percentage of drugs prescribed from essential drugs list or formulary.

\section{Results}

A total of 500 prescriptions were studied where $275(55 \%)$ were females and 225 (45\%) were males. Highest number of patients was in the age group of 15-24 years 196 (39.2\%) followed by the age group of 25-34years (21.4\%) and $<15 y e a r s(17.2 \%)$ and remaining are shown in Table 1. The present study observed that, among the ENT diseases, patient with recurrent tonsillitis $82(16.4 \%)$ was frequently prescribed followed by pharyngitis 75(15\%), CSOM 66 $(13.2 \%)$,allergic rhinitis $60(12 \%)$, ASOM $52(10.4 \%)$, otitis externa $44(8.8 \%)$, sinusitis $36(7.2 \%)$,laryngitis $27(5.4 \%)$ and least were otomycosis, DNS, furunculosis, vestibulitis, candidiasis as in Table 2. Out of total 1535 drugs in 500 encounters, antimicrobials was used in 662 (43.12\%) followed by antihistamines $352(22.93 \%)$, nasal decongestant 331(21.56\%), and analgesic $\&$ antipyretics, others drug like PPI, mucolytic, multivitamins were also prescribed which is shown in Figure 1. Betalactams 263(39.72\%) was commonly prescribed antimicrobial agents. Among them Cefuroximeclavulanate 135 (20.39\%) was frequently prescribed followed by Amoxicillin+ clavulenic acid103 (15.55\%). Another group such as Fluoroquinolones 107 (16.16\%) was also prescribed where ciprofloxacin65 $(9.81 \%)$ was commonly used and least were azithromycin and some topical antifungal drugs. Topical aminoglycosides in the form of gentamicin+ hydrocortisone (steroid) combination 118 $(17.82 \%)$ was frequently used in ENT OPD. Another topical agent was Ciprofloxacin+ dexamithasone103 (15.55\%) combination was used as ear drops in different indication. Among all (662) antimicrobial agents, 420 (63.44\%) drugs were given for oral administration whereas $242(36.55 \%)$ drugs were prescribed in topical formulations as ear drops Table 3. 
The prescription showed that a total of $382(57.70 \%)$ patients received antibacterial monotherapy. Among those who received multiple antibacterial therapy, 246 (37.16\%) received two drug regimen and 34 (5.13\%) received three drug regimen as in Figure 2. Average number of antimicrobials prescribed per patient being 1.32. Different WHO recommended prescribing indicators which as follows: average number of drugs per prescription was 3.07 . No drugs $(0 \%)$ were prescribed in generic form. No injectable medicine $(0 \%)$ was prescribed in this study. Percentage of encounters with an antibiotic prescribed was $100 \%$ and only $28 . .72 \%$ drugs were prescribed from Essential Drug List of Bangladesh 2008.

\section{Table-II}

Demographic profile of the study population

\begin{tabular}{lcc}
\hline Age \& sex distribution & $\begin{array}{c}\text { No. of patients ; } \\
\% \text { of total }\end{array}$ \\
\hline Age & $<15$ & $86(17.2 \%)$ \\
(years) & $15-24$ & $196(39.2 \%)$ \\
& $25-34$ & $107(21.4 \%)$ \\
& $35-44$ & $59(11.8 \%)$ \\
& $45-54$ & $34(6.8 \%)$ \\
Sex & $>54$ & $18(3.6 \%)$ \\
& Female & $275(55 \%)$ \\
& Male & $225(45 \%)$ \\
\hline
\end{tabular}

Table-II

Common ENT diseases

\begin{tabular}{lc}
\hline Diseases & $\begin{array}{c}\text { No. of patients, \% of total } \\
(\mathrm{n}=500)\end{array}$ \\
\hline Recurrent tonsillitis & $82(16.4 \%)$ \\
Pharyngitis & $75(15 \%)$ \\
CSOM & $66(13.2 \%)$ \\
Allergic rhinitis & $60(12 \%)$ \\
ASOM & $52(10.4 \%)$ \\
Otitis externa & $44(8.8 \%)$ \\
Sinusitis & $36(7.2 \%)$ \\
Laryngitis & $27(5.4 \%)$ \\
Otomycosis & $21(4.2 \%)$ \\
DNS & $13(2.6 \%)$ \\
Furunculosis & $11(2.2 \%)$ \\
Vestibulitis & $8(1.6 \%)$ \\
Candidiasis & $5(1 \%)$ \\
\hline
\end{tabular}

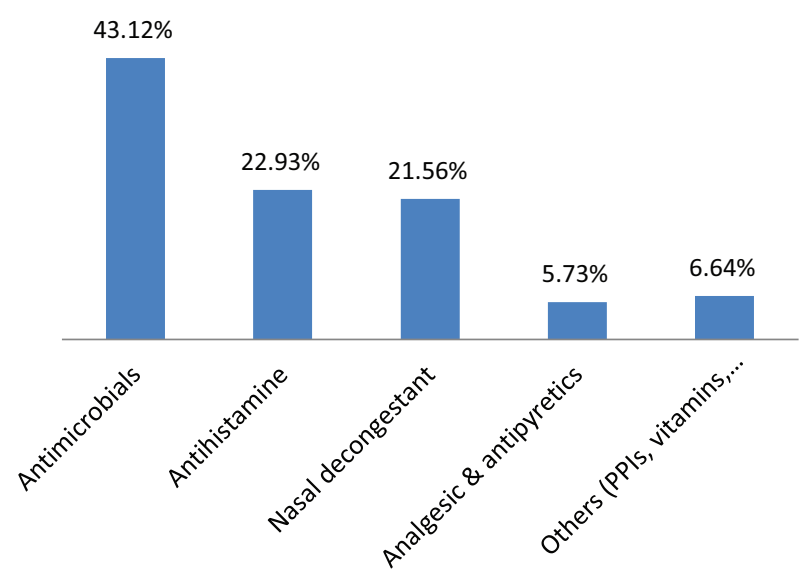

Fig.-1: Commonly prescribed drugs in ENT OPD

Table-III

Distribution of antimicrobial agents

\begin{tabular}{lllc}
\hline $\begin{array}{l}\text { Formulation of } \\
\text { drugs }\end{array}$ & & $\begin{array}{l}\text { Antimicrobials } \\
\mathrm{n}=662\end{array}$ & $\begin{array}{c}\text { No. of drugs ; } \\
\text { of consumption }\end{array}$ \\
\hline Oral & Beta lactams & Cefuroxime +clavulenic acid & $135(20.39 \%)$ \\
& $263(39.72 \%)$ & Amoxicillin+ clavulenic acid & $103(15.55 \%)$ \\
& & Cefixime & $25(3.77 \%)$ \\
& Fluroquinolones & Cefuroxime & $17(2.56 \%)$ \\
& $107(16.16 \%)$ & Ciprofloxacin & $65(9.81 \%)$ \\
& Macrolids 33 (4.98\%) & Aoxifloxacin & $42(6.34 \%)$ \\
Topical & Aminoglycosides+steroid & Gentamicin+Hydrocortisone & $33(4.98 \%)$ \\
(Ear drops) & Fluroquinolones+steroid & Ciprofloxacin+dexamithasone & $118(17.82 \%)$ \\
$242(36.55 \%)$ & Antifungals & Clotrimazole & $21(3.17 \%)$ \\
\hline
\end{tabular}




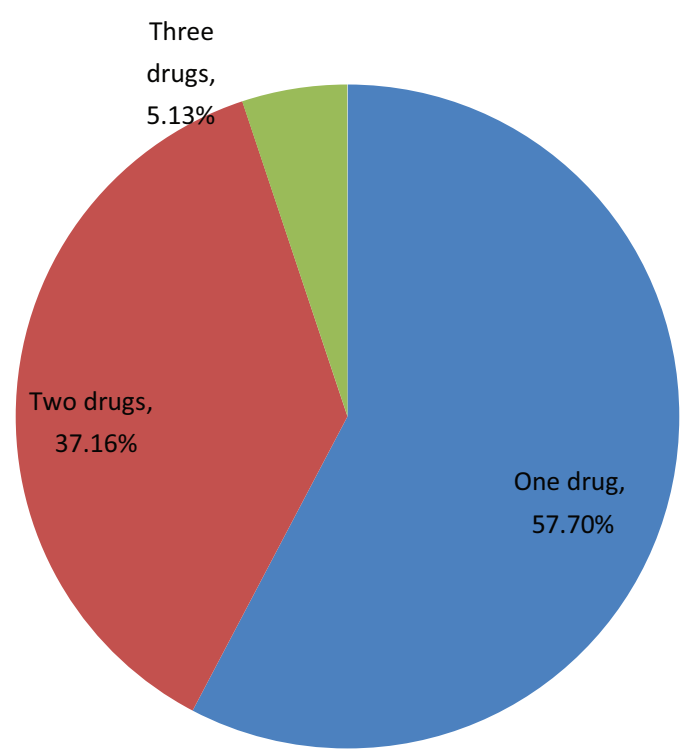

Fig.-2: Frequency of antimicrobials per prescription

\section{Discussion:}

The current trend in prescribing antimicrobial drugs provide a constructive approach in health care delivery system which ensure in solving problems arising from misuse of multiple antibiotic use. The central priority of health care system is providing the right medicine to the right people at the right time. ${ }^{5}$

A total number of 500 prescriptions were analysed and the demographic data showed that females 275 (55\%) were more suffering from ENT infections than males 225 (45\%) during our study period. This is similar with another study done by Naik et al. ${ }^{9}$ In contrast, another study reveals that male were more than their female counterparts ${ }^{3,4,6}$. The maximum ENT infections are more prevalent in young adults in the age group of $15-24$ years (39.2\%) followed by $25-34$ years $(21.4 \%),<15 y e a r s$ patients $(17.2 \%)$ and lowest percentages were in geriatric group. This similar finding were reported in previous studies $4,6,10$ In our study, the most common diseases reported was recurrent tonsillitis $82(16.4 \%)$ followed by pharyngitis 75 (15\%), CSOM 66 (13.2\%) whereas Bhat et al. reported that the most common infection they noticed was CSOM. ${ }^{4}$

Out of total 1535 drugs in 500 prescriptions in our study, most commonly prescribed categories of antimicrobials were â-lactams 263 (39.72\%) followed by Fluroquinolones 107 (16.16\%), macrolides 33 (4.98\%).In a study conducted by Pal et al. was observed that â-lactams was most common, followed by macrolids and quinolone whereas in a study conducted by Khan et al. it was found that most commonly used was âlactams, followed by aminoglycosides and macrolides. ${ }^{3,1}$ Among the cephalosporins most frequently used was combination of cefuroxime and clavulanic acid 135 (20.39\%) and among the penicillins was combination of amoxicillin and clavulanic acid 103 (15.55\%). Cephalosporins are broad spectrum antimicrobials effective against vast majority of organisms, have convenient dosing schedules and have fewer adverse effects; hence they are being prescribed more frequently. Besides cephalosporins and penicillins, Gentamicin and ciprofloxacin was used as topical ear drops combined with Hydrocortisone and Dexamethasone in $17.82 \% \& 15.55 \%$ patients respectively. Among fluroquinolones, ciprofloxacin was used alone in $65(9.81 \%)$ followed by moxifloxacin $42(6.34 \%)$ and lastly, among macrolides most commonly used was azithromycin 33 (4.98\%) patients in our study. This percentage value is not comparable to other studies because of different study populations.

The antimicrobial drugs in majority of the patients were administered orally $(63.44 \%)$ followed by topical administration (36.55\%). Since patients were enrolled from OPD the number of drugs prescribed by oral route was higher rather injection prescribed was nil. This was similar to other studies where majority of antimicrobials were given by oral route. $3,6,10$

In our study $57.70 \%$ patients received antimicrobial monotherapy, whereas $42.29 \%$ were on multiple antimicrobial drug therapy which was similar with other studies. ${ }^{3,10}$.The average number of antimicrobials per encounter was 1.32 . In similar study conducted by Khan et al. the average use of antimicrobials per patient was $1.38 .{ }^{1}$ Other studies done by Pal et al. and Beg et al. average number of antimicrobials per prescription was found to be 1.49 and 1.64 , respectively. ${ }^{3.11}$ The percentage of antimicrobials per encounter was $100 \%$. Majority of the antimicrobials were prescribed 
emperically on the basis of clinical experience of the physicians. Without doing culture and sensitivity test such a high number of prescriptions with multiple antimicrobial therapy is not authentic and rational. Assessing rationality, number of antimicrobials per prescription should be kept low to prevent antimicrobial resistance, drug interactions, adverse effects and increased cost of treatment.

Among the concomitant medications most commonly prescribed drugs were antihistamines $(22.93 \%)$, nasal decongestant $(21.56 \%)$, analgesics $(5.73 \%)$ and others $(6.64 \%)$ drugs like anti ulcer drugs, vitamins, mucolytic, corticosteroids. They were used to provide symptomatic relief for the various ailments.

The average number of drugs per prescription is important in ENT as polypharmacy and misuse of drugs is common. Total 1535 drugs were prescribed for 500 patient making the overall average number of drugs per encounter was 3.07 which is consistent with other studies $^{4,6,12}$ whereas the lower values found were Brazil $2.4^{13}$ and Nepal 2.91 $1^{14}$.Our study result was above WHO recommended value $(<2) .{ }^{15}$ which may have chances into polypharmacy. So it is preferable to keep the average number of drugs per prescription as low as possible.

It was found that all the antimicrobial agents were prescribed by their brand names (100\%), which was not in accordance with the WHO guidelines as rational prescribing that requires generic prescriptions which may reduce overall expenditure on drugs, particularly on newer antimicrobials and avoiding medication errors. This is concordance with other study where brand name prescribing was more. ${ }^{3}$ Regarding drug prescribing from essential drug list EDL, our observation was that, out of total 1535 drugs, only 441 drugs $(28.72 \%)$ in 500 prescriptions were prescribed from the EDL of Bangladesh 2008 which did not meet the standard criteria of prescribing medications from an EDL. Format of the prescriptions with respect to dose, duration, frequency was complete in $100 \%$ of the prescriptions.

On the basis of our observation, it may be mentioned that overall current trend in antimicrobials in ENT OPD was good which may be considered as an effort to improve the quality of health care services.

\section{Conclusions}

All the patients presenting with ENT infections were prescribed antibiotics either single or in combination form. Cefuroxime and clavulanic acid combination was the most commonly prescribed antibiotic in our study. The average number of drugs per prescription was slightly higher than recommended by WHO. Generic name was ignored and list of essential drugs was followed partially. Prescription of drugs in injection form was nil. A further prospective drug utilization study is suggested to improve rational prescribing practice by following appropriate guidelines.

\section{References :}

1. Khan FA, Nizamuddin S, Salman MT. Patterns of prescription of antimicrobial agents in the Department of Otorhinolaryngology in a tertiary care teaching hospital. Afr J Pharm Pharmacol, 2011; 5: 1732-1738.

2. Vanitha M, Vineela M, P BRK, Sandeep P. Prescribing Pattern of Antibiotics in Patients Attending ENT opd in a Tertiary Care Hospital. IOSR Journal of Dental and Medical Sciences (IOSR-JDMS). 2017;16(9):303

3. Pal A, Bhowmick S, Basu J, Chattopadhyay R, Paul SS, Chattopadhyay S. Study on prescribing pattern of antimicrobials in ENT department of a tertiary care teaching hospital in Bihar,India. World Journal of Pharmaceutical Research. 2015; Volume 4, Issue 8, 1839-1852.

4. Bhat GMN, Holla R, Shrinath D. Kamath P. A study of prescription pattern in the drug therapy of ear, nose, and throat infections at a tertiary care hospital in Mangalore. Int J Basic Clin Pharmacol. 2015 Aug;4(4):686-690.

5. Gupta SP, Poudel S, Gupta AP, Bashyal B, Baskota $\mathrm{B}$, Sah AK, et al. A prospective cross-sectional study on prescribing pattern of antibiotics on patients suffering from ENT infections in tertiary care hospital, Pokhara, Nepal. Int J Basic Clin Pharmacol. 2017 Oct;6(10):2303-2310

6. Y KK, Cheekavolu C, Obulesu G. Drug utilization pattern in ENT OPD of government tertiary care teaching hospital in Raigarh. Int J Otorhinolaryngol Head Neck Surg. 2017 Oct;3(4):1042-1045

7. N. B Guru Prasad, Dhananjay K, C. H Rajasekhar, D Rajesh, A. Y Raghavendra, K Vinodraj et al. A study 
of prescription pattern of antimicrobial usage in ear,nose and throat infections of a rural teaching hospital. Journal of Evolution of Medical and Dental Sciences. 2014; Vol.3(60):13407-13414.

8. Kancherla D, M V Satya Sai, HJ Gayathri Devi,Sharma S. A study on prescribing pattern of antibiotics in respiratory tract infections in a tertiary care center. International Journal of Recent Scientific Research. 2015; Vol. 6, Issue, 6, pp.4558-4563.

9. Naik HG, Khanwelkar CC, Kolur A, Desai R, Gidamudi S. Drug Utilization Study on Antibiotics Use in the Upper Respiratory Tract Infection. International Journal of Recent Trends in Science And Technology.2014; Volume 10, Issue 2, pp 299302.

10. Padwal SL, Kulkarni MD, Deshmukh VS, Patil JR, Jadhav SS, Jadhav AD. National Journal of Physiology, Pharmacy and Pharmacology. 2015;Vol $5(3): 212-216$.

11. Beg MA, Bawa S, Dutta S, Anjoom M, Vishal S. Study of antimicrobial prescribing pattern in a tertiary care teaching hospital at Dehradun, Uttarakhand, India - A tool to teach clinical pharmacology to MBBS students. Int $\mathrm{J}$ Basic Clin Pharmacol. 2016 Dec;5(6):2444-2448.

12. Suman RK, Kumar R, Mohanty IR, Deshmukh YA. Assessment of Drug Usage Pattern of Antibiotics Used in ENT OPD of Tertiary Care Teaching Hospital. Int J Health Sci Res. 2015; 5(9):290-297

13. Acurcio FA, Perini E, Magalhães SMS, Terceiro LGV, Filho JMV, Coutinho KEAO M et al. Analysis of medical prescriptions dispensed at health centers in Belo Horizonte, Minas Gerais, Brazil. Cad. Saúde Pública, Rio de Janeiro. 2004 jan-fev;20(1):72-79

14. Alam K, Mishra P, Prabhu M, Shankar PR, Palaian $\mathrm{S}$, Bhandari RB, et al. A study on rational drug prescribing and dispensing in outpatients in a tertiary care teaching hospital of Western Nepal. Kathmandu University medical journal(KUMJ). 2006;4(4), pp.436-443.

15. Summoro TS, Gidebo KD, Kanche ZZ, Woticha EW. Evaluation of trends of drug-prescribing patterns based on WHO prescribing indicators at outpatient departments of four hospitals in southern Ethiopia. Drug Design, Development and Therapy 2015:9 4551-4557. 